\title{
Cerebral occlusive vasculopathy in systemic lupus erythematosus and speculation on the part played by complement
}

\author{
Ali Hammad, Yoshiaki Tsukada, Nelson Torre
}

\begin{abstract}
A 35 year old woman with systemic lupus erythematosus and hypocomplementenaemia presented with new onset seizures and subsequently died. At necropsy, widespread microinfarctions of the cerebral cortex were found to be predominantly due to the formation of leucoaggregates within small blood vessels without any vasculitis.
\end{abstract}

Despite recent advances in the understanding of the pathophysiology of systemic lupus erythematosus (SLE), the pathogenesis of some complications of SLE remains enigmatic and, in some instances, more than one mechanism may be responsible for a similar complication. Considerable controversy surrounds the pathogenesis of central nervous system disease in SLE. We report here an unusual case in an attempt to explain a mechanism that may play a part in central nervous system disease in SLE.

\section{Case report}

A 35 year old woman with a history of SLE was admitted to the Sisters of Charity Hospital with rapidly progressive shortness of breath.

She had been in good health until three years before this admission when she was admitted to hospital for polyarthritis. Pertinent laboratory data at that time were as follows: haemoglobin $101 \mathrm{~g} / \mathrm{l}$, packed cell volume $30 \cdot 3$, platelets $135 \times 10^{9} / 1$, microscopic haematuria, $2+$ proteinuria, positive antinuclear antibody screen (titre 280, homogeneous), positive antibodies to double stranded DNA (titre 1/320), total complement $22 \%$ of reference (normal 51-150\%), C3 0.29 g/l (normal 0.7-1.76 g/l), C4 $0.05 \mathrm{~g} / \mathrm{l}$ (normal $0 \cdot 18-0.45 \mathrm{~g} / \mathrm{l}$ ). Serum electrolytes, blood urea nitrogen, and creatinine were normal. Rheumatoid factor and anticardiolipin antibodies were absent. A diagnosis of SLE was

The patient also developed immune thrombocytopenia and her platelet counts reached a minimum of $39 \times 10^{9} / 1$. High dose prednisone resulted in an amelioration of symptoms and an increase of the platelet count to normal levels. A renal biopsy, performed two months later, showed a mild and sparse sclerosing and proliferative glomerulonephritis. Proteinuria was quantified at $2 \cdot 2 \mathrm{~g} /$ day. She continued to have low C3 and C4 levels and developed a vasculitic rash on her hands and feet. She was then treated with azathioprine and prednisone for approximately seven months with improvement.

Subsequently, she developed maxillary sinusitis requiring admission to hospital and treatment with antibiotics. At that time, azathioprine was stopped and prednisone was decreased to $20 \mathrm{mg} /$ day. Two months before this admission she developed a Mycobacterium chelonei infection of the skin of the left upper arm and was admitted to the hospital for intravenous treatment with antibiotics. At the time of this admission she was receiving prednisone (20 mg/day).

On examination she was alert and oriented but obviously dyspneic. Vital signs were as follows: blood pressure $150 / 70 \mathrm{mmHg}$, temperature $37^{\circ} \mathrm{C}$, pulse 120 beats/minute, respiratory rate 32 breaths/minute. Jugular venous distention was noted. An S4 gallop was heard. Her lungs were clear and her abdomen was normal. No focal neurological deficits were noted. Serum sodium, potassium and glucose were normal. Creatinine was $240 \mu \mathrm{mol} / \mathrm{l}$ and lactate dehydrogenase was $1488 \mathrm{U} / \mathrm{l}$. Liver enzymes were normal. Her white blood cell count was $12 \cdot 7 \times 10^{9} / 1$, haemoglobin was $100 \mathrm{~g} / \mathrm{l}$, packed cell volume was 31 . Urine analysis showed a specific gravity of $1 \cdot 023, \mathrm{pH} 5$, protein $3+$, red blood cell count 10-20/high powered field, white blood cell count $2-5 /$ high powered field, bacteria $1+$, and no leucocyte esterase or glucose. The coagulation profile was normal. Blood, urine, and sputum specimens were obtained for culture. An electrocardiogram showed sinus tachycardia with a nonspecific ST abnormality. Echocardiography showed diffuse hypokinesis, a left ventricular ejection fraction of $25-30 \%$, and a moderate sized thrombus in the left ventricle. Shortly after presentation, she developed intermittent tonic clonic seizure activity. Intravenous heparin for possible cerebral emboli and high dose parenteral corticosteroids for possible vasculitis were begun. She developed cardiac arrest from which she was promptly and successfully resuscitated, but she then remained comatose and hypotensive. Arterial blood gas determinations showed a severe and persistent metabolic acidosis. Empirical treatment with antibiotics was begun with cefoxitin, amikacin, and erythromycin. A computed tomography scan of the brain showed multiple cortical infarctions without intracranial haemorrhage. The patient then developed acute renal failure with anuria. She finally succumbed to her multisystem disease less than 48 hours after admission. Cultures of blood, urine, and sputum remained negative.

At necropsy, anasarca was noted. The heart showed concentric hypertrophy but no mural thrombus was found. The lungs showed severe made. 
passive congestion. No vasculitis was found in the heart or the lungs. The kidneys were shrunken and showed severe glomerulosclerosis and crescentic glomerulonephritis. The pathology of the brain was important. Multiple small cortical infarctions were seen scattered throughout the cerebral hemispheres (fig 1). Microscopic examination showed these infarctions to be one to two days old with acute ischaemic changes of the neurones and polymorphonuclear infiltration. A few older microscopic infarcts were present. The vessels in the areas of infarction were normal in appearance and free of any vasculitic process. A main pathological process appeared to be occlusion of these small vessels by aggregates of leucocytes (fig 2). Occlusion of small vascular lumens by fibrin thrombi was

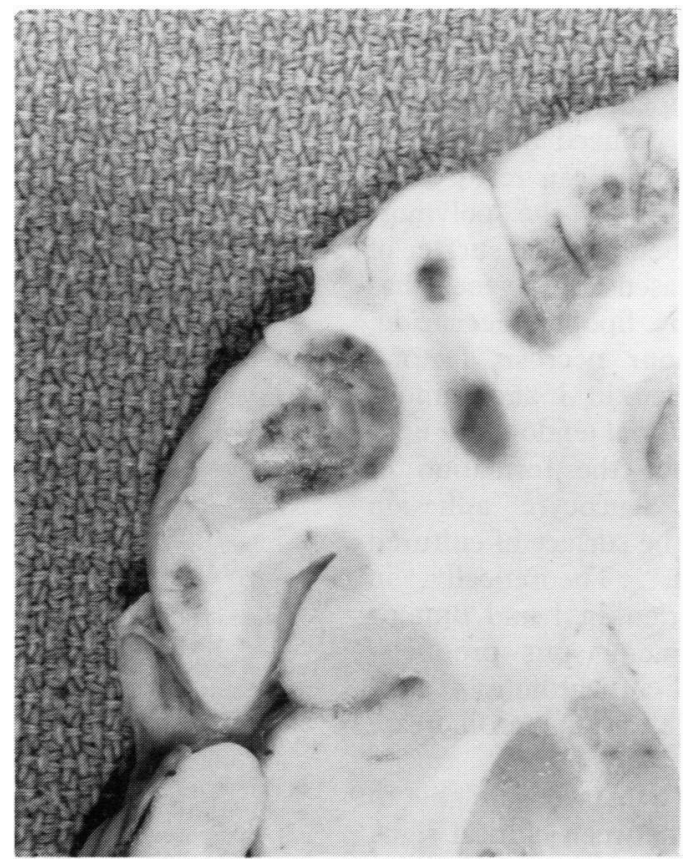

Figure 1 Photograph of the frontal lobe of the cerebrum showing multiple recent cortical infarcts.

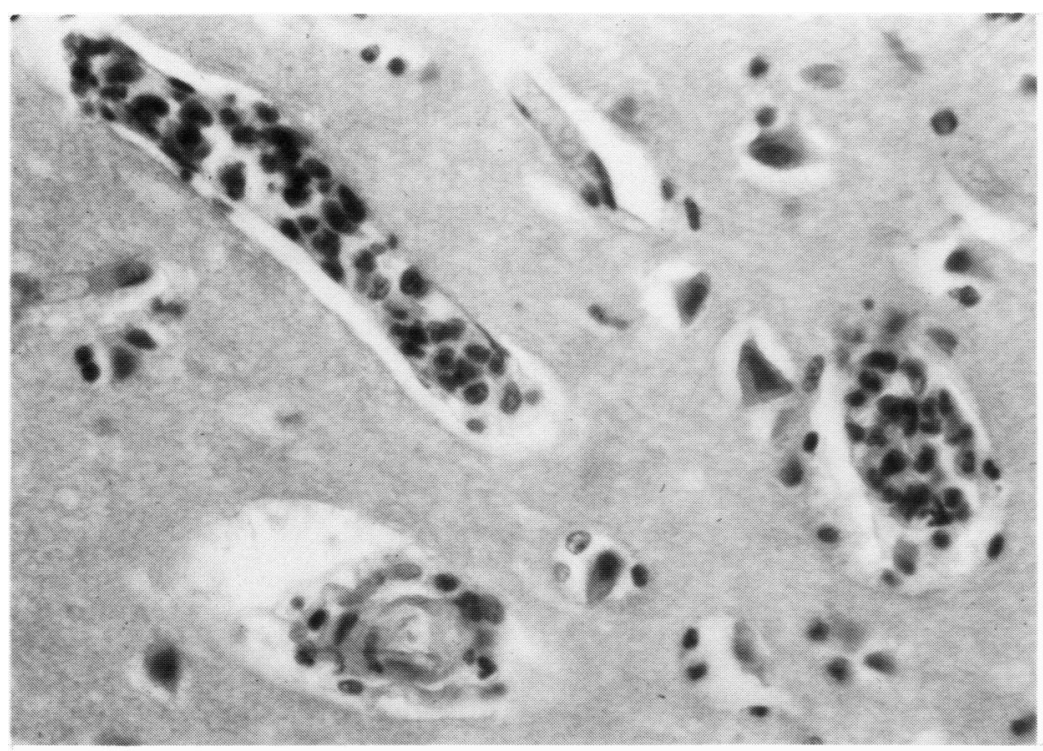

Figure 2 Occlusion of small blood vessels with leucocyte aggregates, and neurones with acute anoxic changes (haematoxylin and eosin stain).

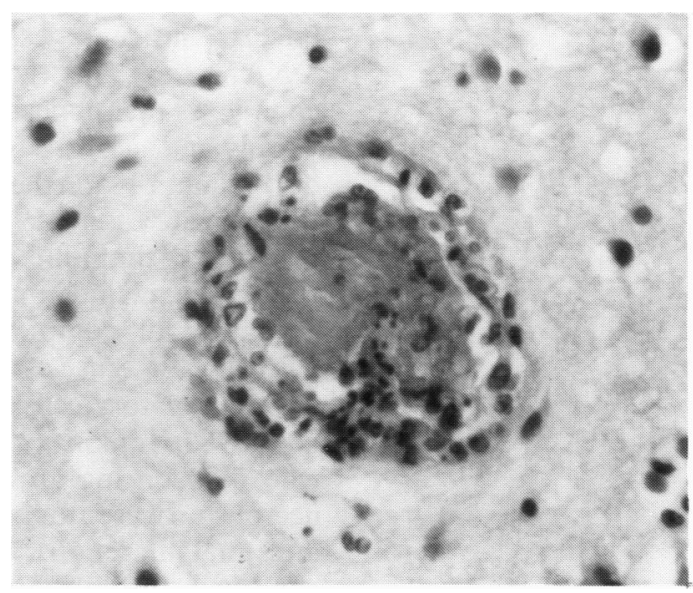

Figure 3 Fibrin microthrombus within a small blood vessel (haematoxylin and eosin stain).

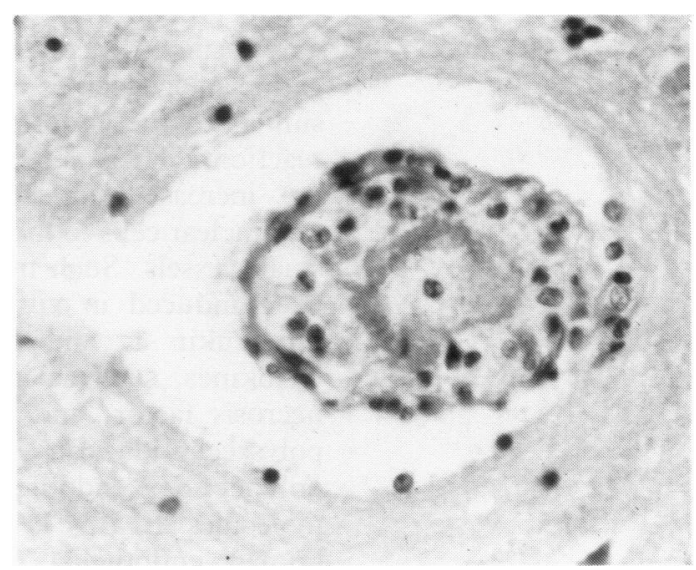

Figure 4 Leucocytes in a Virchow-Robin space (haematoxylin and eosin stain).

rare (fig 3). A few small vessels showed VirchowRobin spaces filled with leucocytes, representing extension of leucocytes from the subarachnoid space (fig 4).

\section{Discussion}

The clinical manifestations of central nervous system disease in SLE are protean. They include seizures, cranial nerve disorders, hemiparesis, paraparesis, peripheral neuropathy, movement disorders, meningeal signs, and autonomic disorders. ' Psychiatric manifestations and disorders of mental functions are also well described. ${ }^{2}$ The pathogenesis of central nervous system disease in SLE, however, remains ill defined. In one necropsy series, the major neuropathological findings included vasculopathy, infarction, haemorrhage, and infection. ${ }^{3}$ Vasculopathy was found in $65 \%$ of the studied cases and was further subclassified as vascular hyalination, perivascular inflammation, endothelial proliferation, thrombosis, and, rarely, vasculitis. Antiphospholipid antibodies, which may be present in up to $44 \%$ of patients with SLE, have also been associated with an increased propensity towards thrombosis. ${ }^{4}$ Complement probably has an important part to play in vascular injury in SLE. The role of immune complexes in complement activation is well established. Autoantibodies against 
nuclear antigens, especially double stranded DNA, result in the formation of immune complexes that deposit within the walls of small vessels and activate complement at the site of deposition. ${ }^{5}$ Nevertheless, some lupus patients have small vessel occlusive disease without any evidence of immune complex deposition. This could be explained on the basis of intravascular activation of complement via the alternative pathway as a cause of activation and aggregation of inflammatory cells in SLE. ${ }^{\circ}$

In 1988 Hopkins et al ${ }^{7}$ reported two patients who died during an acute lupus flare and, at necropsy, were found to have cerebral and intestinal infarctions. Microscopy failed to show any fibrin thrombi or vasculitis. Many vessels, however, were found to be occluded by aggregates of neutrophils. They suggested that circulating anaphylatoxins, $\mathrm{C} 3 \mathrm{a}$ and $\mathrm{C5a}$, products of intravascular complement activation, may result in occlusive vasculopathy by aggregation of leucocytes and platelets in a manner similar to the endotoxin induced Shwartzman reaction. ${ }^{8}$ These leucothrombi can result from the increased adhesiveness of the polymorphonuclear cells to the subendothelial surface of small vessels. Such increased adhesiveness has been induced in vitro by lipopolysaccharide, interleukin 1, and tumour necrosis factor. ${ }^{9}$ Cytokines, such as interleukin 1 and tumour necrosis factor, and bacterial endotoxin lipopolysaccharide can induce the formation of polypeptide endothelial leucocyte adhesion molecules (ELAM 1) on the surface of cultured human endothelial cells. ${ }^{10}$ Theoretically, in SLE, in addition to interleukin 1 and tumour necrosis factor, complement split products, immune complexes, and anticardiolipin antibodies may be responsible for such an increase in vascular adhesiveness. ${ }^{7}$

In the patient reported here, we were able to show a good correlation between clinical signs (seizures and coma) and pathological findings (diffuse cortical infarcts of various ages). Microscopically, the predominant lesion was that of occlusive vasculopathy due to aggregation of leucocytes within vessels subtending infarctions (fig 2). The patient had low $\mathrm{CH} 50, \mathrm{C} 3$, and $\mathrm{C} 4$ levels throughout the course of her illness. This probably represented continually active disease and ongoing complement activation with inflammation. Complement activation products and endogenous mediators of inflammation could have resulted in increased adhesiveness of the cerebral vascular endothelium by induction of ELAM-1 like molecules with subsequent leucothrombus formation leading to cerebral infarction. It is noteworthy that anticardiolipin antibodies were absent in this patient.

We have thus reported a patient with SLE with major central nervous system disease resulting in death. The pathological findings suggest that the mechanism of infarction was related to leucothrombus formation. Further studies are needed to elucidate the pathogenetic mechanism involved.

1 Johnson R T, Richardson E P. The neurological manifestations of systemic lupus erythematosus. Medicine (Baltimore) 1968; 47: 347-69.

2 Feinglass E J, Arnett F C, Dorsch C A, Zizic T M, Stevens $M$ B. Neuropsychiatric manifestations of systemic lupus erythematosus: diagnosis, clinical spectrum, and relation ship to other features of the disease. Medicine (Baltimore) ship to other feat
1976; 55: 323-39.

3 Ellis S G, Verity M A. Central nervous system involvement in systemic lupus erythematosus: a review of neuropathologic findings in 57 cases, 1956-1977. Semin Arthritis Rheum $1979 ; 8: 212-21$.

4 Love P E, Santoro S A. Antiphospholipid antibodies: anticardiolipin and the lupus anticoagulant in systemic lupus erythematosus (SLE) and in non-SLE disorders. Ann Intern Med 1990; 112: 682-98.

5 Fauci T Y, Haynes B F, Katz P. The spectrum of vasculitis. Ann Intern Med 1978; 89: 660-76.

6 Abramson S B, Weissmann G. Complement split products and the pathogenesis of SLE. Hosp Prac [Of] 1988; 15: a5-56.

7 Hopkins P, Belmont H M, Buyon J, Philips M, Weissmann $G$, Abramson S B. Increased levels of plasma anaphylatoxins in systemic lupus erythematosus predict flares of the in systemic lupus erythematosus predict flares of the disease and may elicit vascular in
Arthritis Rheum 1988; 31: 632-41.

8 Shwartzman G. Phenomenon of local tissue reactivity. New York: Paul Hoeber, 1937.

9 Pohlman T H, Stanness K A, Beatty P G, Ochs H D, Harlan $J M$. An endothelial cell surface factor(s) induced in vitro by lipopolysaccharide, interleukin 1 , and tumor necrosis factor alpha increases neutrophil adherence by a CDw18dependent mechanism. F Immunol 1986; 136: 4548-53.

10 Bevilacqua M P, Pober J S, Mendrick D A, Cotran R S, Gimbrone M A Jr. Identification of an inducible endothelialleukocyte adhesion molecule. Proc Natl Acad Sci USA 1987; 84: 9238-42. 